\title{
Dynamic recrystallization behavior of the Ta-containing TiAl alloy in isothermal deformation
}

\author{
Y.Y. Luo *,X.N.Mao, H.Y.Yang, Y.F.Yin, Z.Z.Zhao,D.Z.Guo \\ Northwest Institute for Nonferrous Metal Research, Xi'an 710016, China
}

${ }^{*}$ Corresponding author. Tel: +86-29-86231078 ; fax: +86-29-86360416; E-mail addresses: grass8180@126.com (Y.Y.Luo)

\begin{abstract}
The dynamic recrystallization behavior of as-cast Ti-46.5Al-3 Ta-2Cr- $0.2 \mathrm{~W}$ alloy during isothermal compression process with nominal deformation of $50 \%$ and strain rates from $0.01 \mathrm{~s}-1$ to $1 \mathrm{~s}-1$ was investigated by electron microscopy. The results showed that the deformation mechanism of this alloy can be concluded as grain boundary sliding and mechanical twins, which induce the final dynamic recrystallization. The phase boundary bulging was found to be the major nucleation mechanism responsible for the lamellar globularization and the formation of recrystallized $\gamma$ grains inside the lamellar colony under the high strain rate. The recrystallized $\gamma$ grains induced by the twinning is the main mechanism for refining $\alpha_{2}$ lamellar microstructures under low strain rate.
\end{abstract}

Key words: Intermetallic alloys and compounds; Microstructure; Recrystallization

\section{Introduction}

Now it is well known that the processes such as dynamic recovery (DRV), dynamic recrystallization (DRX), postdynamic recrystallization (MDRX), static recovery (SRV), static recrystallization (SRX), and grain growth may each affect the final microstructure of metals that is produced during thermomechanical processing [1]. Especially, DRX can take place during deformation when plastic deformation is carried out at high enough temperatures and many DRX mechanisms have been proposed [2 4]. Grain boundary mobility and driving forces are the key factors for the occurrence of DRX. Therefore, the appearances of the DRX are roughly classified into two major types [5 6]: the discontinuous and continuous dynamic recrystallization (DDRX and CDRX) based on the relative importance of grain boundary bulging (BLG) and subgrain rotation (SGR) nucleation. During continuous 
dynamic recrystallization, sub-boundaries form as deformation proceeds, and high-angle boundaries may develop by progressive misorientation of sub-boundaries. By contrast, discontinuous dynamic recrystallization (DDRX) is characterized by a rapid migration of grain boundaries between dislocation-free nuclei and deformed grains [7 9]. The DDRX usually takes place in metals with medium stacking fault energy (SFE), and the CDRX usually takes place in the metals with the high SFE under certain conditions. Many researcher cared about the DDRX in based alloys with high SFE, however only a few investigators have paid attention to that how the CDRX and DDRX form respectively with the strain rates and how they play a role in isothermal deformation mechanism by hot compression tests in TiAl based alloys.

The objective of the present study was to (i) determine whether CDRX or DDRX occurs during isothermal deformation conditions of full lamellar material studied here at the strain rates of $1,0.5$ and $0.01 \mathrm{~s}-1$. (ii) make substantial progress to understand the formation mechanism of CDRX in the hot deformation of the Ti-46.5Al-3Ta-2Cr-0.2W alloy.

\section{Experimental Procedures}

The ingot of the alloy with a nomial composition (in atom fraction) of Ti-46.5Al-3Ta-2Cr- $0.2 \mathrm{~W}$ was produced by vacuum arc melting. The cylindrical ingot with a diameter of $30 \mathrm{~mm}$ and length of $130 \mathrm{~mm}$ was then homogenized at $1173 \mathrm{~K}$ for $10 \mathrm{~h}$. Cylindrical specimens with a diameter of $8 \mathrm{~mm}$ and a height of $12 \mathrm{~mm}$ for compression test were machined from the homogenized ingot by electro-erosion. The high temperature compression tests were conducted in Gleeble 3800 at $1473 \mathrm{~K}$ with the strain rates of $1,0.5$ and $0.01 \mathrm{~s}-1$ and the specimens were resistance heated to compression temperatures by thermocouples feedback at the rate of $5^{\circ} \mathrm{C} / \mathrm{s}$ and held for $3 \mathrm{~min}$ before hot compression so as to ensure homogenized temperature. After isothermal compression, the specimens were quenched in air. Thin foils for transmission electron microscopy (TEM) observation were also cut parallel to the compression axis from the deformed specimens. TEM observation was performed in a EM-200GX operated at 200kV. Crystal structures of the cast Ti46.5Al-3Ta-2Cr-0.2W alloy were characterized using XRD with $\mathrm{Cu} \mathrm{K}_{\alpha}$ radiation. 


\section{Results and discussion}

\subsection{Initial microstructure}

The initial microstructure of cast Ti-46.5Al-3Ta-2Cr- $0.2 \mathrm{~W}$ alloy was a nearly fully lamellar structure composed of $\gamma+\alpha_{2}$ lamellae as shown in Fig.1a and b. The nano-twins in the laths arranged quite regularly and the lamellar boundaries were clear and straight in the original alloy. Moreover, many ordered fault ribbons were discovered in the $\gamma$ lamellae, besides a small amount of dislocations that might form in the specimen.
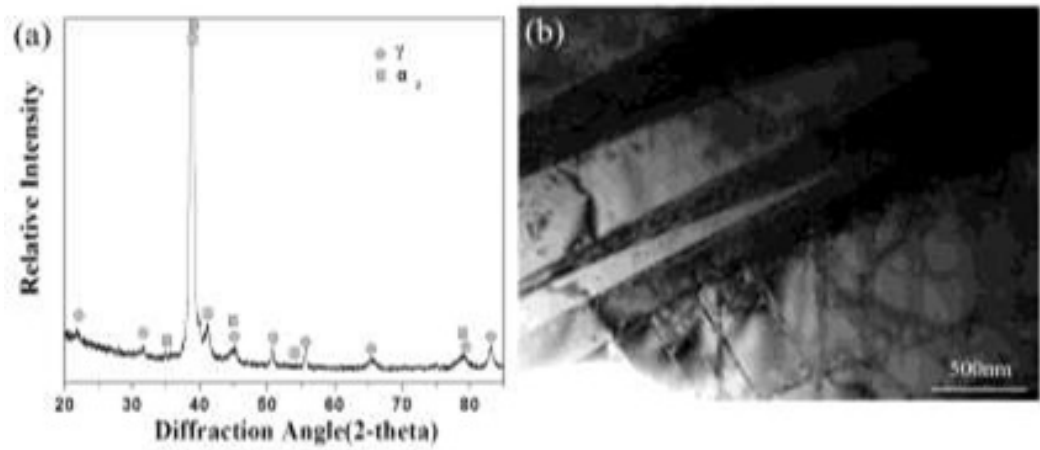

Fig.1 The microstructure of the cast Ti-46Al-3Ta-2Cr-0.2W alloy homogenized at 1473K/10h (a)XRD (b) nano-twins in the lath.

\subsection{Microstructures at high strain rate}

The microstructure after high temperature deformation at $1473 \mathrm{~K}$ with $1 \mathrm{~s}^{-1}$ was composed mainly of $\alpha_{2} / \gamma$ lamella and recrystallized grains (Fig.2). It is interesting to note that a high density of dislocations and a great number of formed deformation boundaries (subgrain boundaries) is observed at all the strain rates and the strain levels as shown in Fig.2a. High density of dislocation stacks in the boundary of lamellar structure, which can provide enough driving force for DRX[10]. Therefore, the lamellae are fragmented and 
spheroidized, and finally transformed to be the equiaxed DRX grains with fine grain sizes in the range $0.2 \sim 1 \mu \mathrm{m}$.Seshacharyulu et.al[11] thought that the globularization process may also be regarded as a type of dynamic recrystallization since it also involves two competitive processed via, rate of nucleation and rate of migration. It can be considered as the $\gamma_{\mathrm{R}}$ grains in this alloy, so the globularized microstructure consisted of dynamically recrystallized grains, which contained a high density of dislocations and networks, plus equiaxed dislocation-free grain. Besides, this recrystallization initiate by subgrain rotation is currently recognized as a common DRX mechanism in the hot-working of metals, and is referral to as "CDRX" in the present alloy because the grain boundary migration did not occur in this stage. The other type of DRX is shown in Fig.2b at center of the specimen with the same strain rate. The fine lamella merged with the others by the local strain-induced bulging and migration of grain boundaries. During thermal compression, the ledges in the $\gamma / \gamma$ and $\gamma / \alpha_{2}$ interfaces would move because of gliding of interfacial dislocations and twinning, and the DRX grains would grow into $\gamma$ phase with higher deformation energy and grow normal to the interfaces because of the climbing and cross-slipping of the interfacial dislocations.

The microstructural processes of the new grain formation during DRX are roughly classified into two major types [12 13]. One is due to the bulging of grain boundaries, in which new grains are formed by the local strain-induced migration of grain boundaries (as shown in Fig.2b). At this stage, the alloy is characterized by a "necklace" structure, whereby nuclei grow at the expense of existing grains. The other is due to the nucleation and growth of new grains, in which nuclear of new grains are formed in heavily deformed regions neighboring the grain boundaries (as shown in Fig.2c). Normally, both recrystallization and grain growth occur more rapidly farther from the stoichiometric composition, due to faster diffusion kinetics.

In short, at the high strain rate, the preserved substructures in some coarse grains are found significantly facilitate the strain-induced subgrain boundaries increasingly transforming to higher-angle boundaries by continuous deformation, which promotes the occurrence of the CDRX in TiAl alloy. Moreover, in some fine grains, the formation of DRX relied on the merged grain and the bulging of grain boundaries and it was more inclined to form the DDRX. This discovery probably indicates that the formation mechanisms of the DRX have respect to the width of the original lamella. 


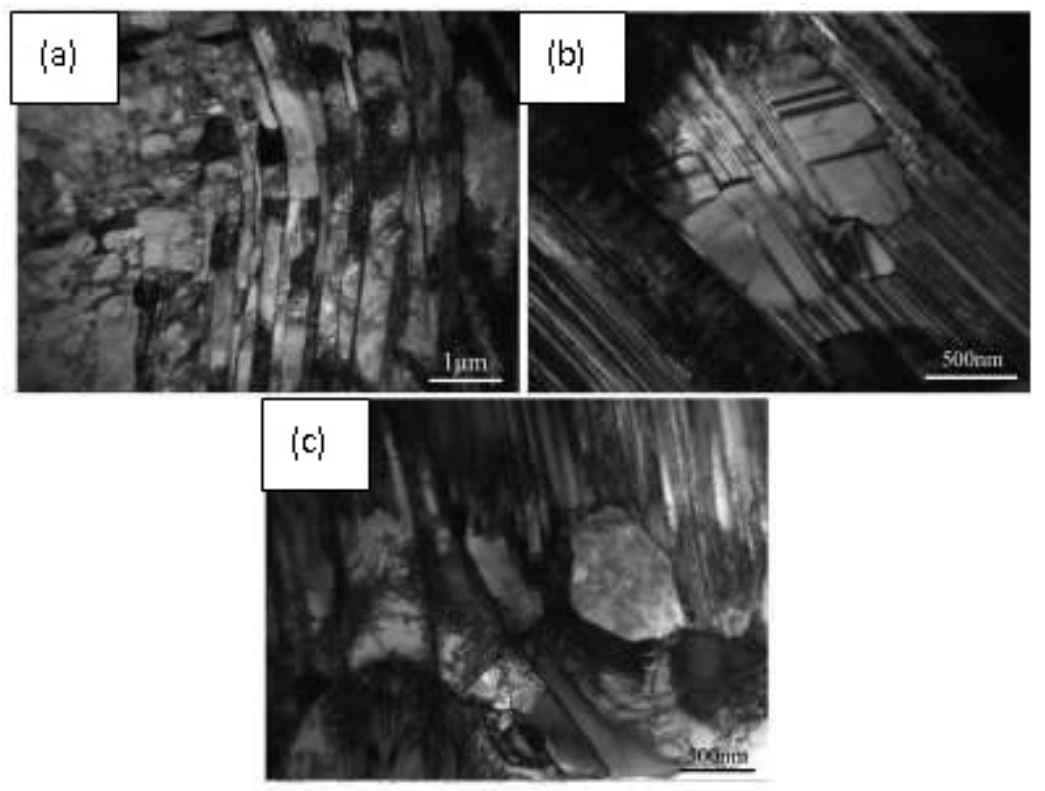

Fig.2. TEM photographs with the strain rate of $1 \mathrm{~s}-1$ for Ti-46.5Al-3Ta-2Cr-0.2W alloy:(a) fragmentation and spheroidization of $\alpha 2$ lamellae

and typical DRX grains (b) The grains with bulging (c) new recrystallized gamma grains at grain boundary.

\subsection{Microstructures at low strain rate}

The microstructure after high temperature deformation at $1473 \mathrm{~K}$ with $0.5 \mathrm{~s}-1$ was composed mainly of $\alpha_{2} / \gamma$ lamella and recrystallized grains (Fig.3). The fine equiaxed and white grains essentially free of dislocations in Fig.3a were DRX grains and the SAD patterns showed that the $\gamma_{R}$ have the following crystallographic relationships with the $\alpha_{2}:<110>\gamma_{R} / /\{1120] \alpha_{2}$ and $\{111\}$ $\gamma_{\mathrm{R}} / /(0001) \alpha_{2}$. During CDRX, sub-boundaries form as deformation proceeds and high-angle boundaries may develop by progressive misorientation of sub-boundaries.Fig. $3 b$ indicates the some ordered twin related variants of $\gamma_{R}$ exist in $\beta$ grain. It is well t the twinning plays a dual role during deformation and it is a "self-stopping" process owing to fragmentation of grains and the twinning 
development is stopped that may disturb the compatibility of deformation between the fragments and restrict the plastic flow of the intermetallic[14]. Furthermore, coherent twin boundaries have long been recognized as effective boundaries to plastic deformation in f.c.c metals. Thus, this twin will provide more nucleation position for DRX in this alloy. Besides, Singh et al [15] reported the Ta addition did not change the overall microstructure, but the twin thickness was reduced in TiAl based alloy and the twin boundaries appear to favor additional shear deformation parallel to an existing boundary. Thus, it is concluded that the Ta addition is beneficial to the deformation of this alloy.

The microstructure with $0.01^{\mathrm{s}-1}$ was composed mainly of fine recrystallized grains at grain boundary (Fig.4a) .Those areas are characterized by a microstructure consisting of black grains plus fine equiaxed containing a high density of dislocations and white grains (and subgrains) essentially free of dislocation. The numerous equiaxed grains could be the result of dynamic recrystallization during air cooling after the compression and belong to the DDRX type. The fine grains which contain dislocations must have recrystallized and subsequently were under gone further working during the compression. Their sizes are in the range $0.2 \sim 0.5 \mu \mathrm{m}$, which are more fine than the ones at the high strain rate. Fig. $4 \mathrm{~b}$ also indicates that the ordered twin related variants of $\gamma_{R}$ exist in one grain, and thin deformation twins can be observed in variants. The SAD showed the relationship between $\gamma_{\mathrm{R}}$ and the $\gamma$ grains. .Kim et al. [16] reported that the low strain rate favors the Ti3Al phase dynamic recrystallization sufficiently The fine recrystallized $\gamma$ grains are more than the ones at the high strain rates clearly in this ally and also finer on the size. 


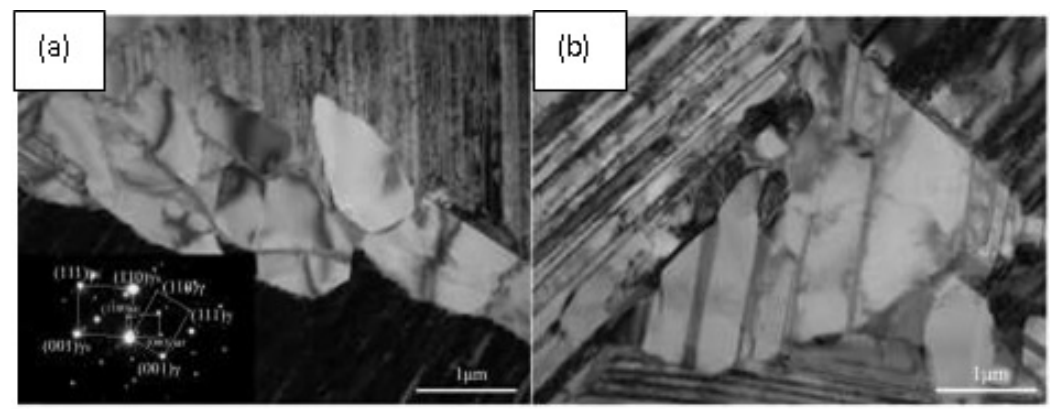

Fig.3 TEM photographs with the strain rate of $0.5 \mathrm{~s}-1$ for Ti-46.5Al-3Ta-2Cr- $0.2 \mathrm{~W}$ alloy (a) new recrystallized grains at the existing grain boundary and the corresponding SAD (b) $\gamma \mathrm{R}$ grains by twining. More stacking faults mean DRX can take place more easily, and twins readily become the nuclei of DRX

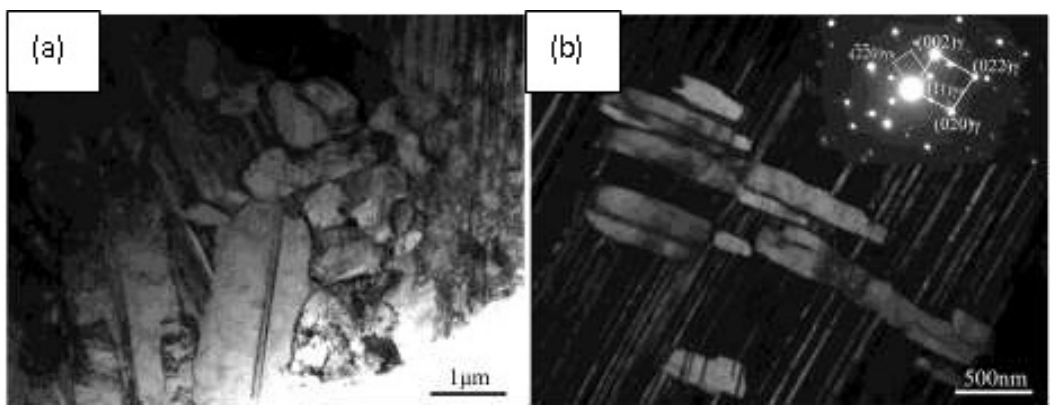

Fig.4 TEM photographs with the strain rate of $0.01 \mathrm{~s}-1$ for Ti-46.5Al-3Ta-2Cr-0.2W alloy (a) new recrystallized gamma grains at grain boundary (b) a continuous $\gamma \mathrm{R}$ grains by twinning and the corresponding SAD.

\section{Conclusions}

The evolution of microstructure and DRX grains with compression strain rates from 10-2 to $1 \mathrm{~s}-1$ in the Ti-46.5Al-3Ta-2Cr$0.2 \mathrm{~W}$ alloy at $1473 \mathrm{~K}$ has been investigated. The recrystallized $\gamma_{\mathrm{R}}$ grains nucleated in the phase boundary when the phase boundary bulging and migration under high strain rate deformation during the thermal compression. With decreasing strain rate, the different $\gamma_{\mathrm{R}}$ grains nucleated inside the $\alpha_{2}$ lamella colony by the twinning and seemed to occur more easily and extensively by easier grain boundary sliding and enough allocated time for migration. 


\section{Acknowledgements}

This research was sponsored by the Natural Science Foundation of China (No.51201138). The author would like to thank

Dr.L.Li and Dr.C.Xue in Northwest Institute for Nonferrous Metal Research for providing the hot compressiom.

\section{References}

[1] S.L.Semiatin D.S.Weaver, R.C.Kramb et al.Metal and Mater Trans A, 35(2)(2004),679-693.

[2] M.A.Stucke, V.K.Vasudevan, D.M.Dimiduk et al. .Mater Sci Eng A , 192/193(1995)111-119.

[3] S.Z. Zhang, C.J. Zhang, Z.X. Du, et al..Mater. Sci. Eng. A 642 (2015) 16-21.

[4]L.Xiang,B.Tang, X.Y.Xue et al.Intermetalics.97(2018):52-57

[5] I.Shimizu.J Struct Geol, 30(7)(2008),899-917

[6] D.G.Cram X.Y.Fang, H.S.Zurob et al. Acta Mater, 60(18)(2012), 6390-6404.

[7] P. Duval. F.Louchet, J.Weiss et al.Mater Sci Eng A, 546(1)(2012),207-211.

[8] Z.C.Liu, J.P.Lin, Y.L.Wang et al.Mater Lett, 58(6)(2004),948-952.

[9] L.Cheng H.Chang, B.Tang et al.J Alloys Compd, 552(5)(2013)363-369.

[10] L.Xiang,B.Tang,X.Y.Xue. et al.Intermetallics.97(2018)52-57.

[11] T. Seshacharyulu S.C.Medeiros, J.T.Morgan et al. Scripta Mater , 41(3)(1999), 283-288.

[12] M.Hasegawa H.Fukutomi .Mater Trans JIM, 43(9)(2002),1183-1186. 